\title{
Quantifying the Allocation of Monograph Funds: An Instance in Practice
}

\section{William McPheron}

This paper describes a formula for distributing monograph funds that was developed at a medium-sized university library by a committee of subject specialists working closely with other bibliographers. Relying on a combination of objective data and professional judgment, the method employs a size-of-literature approach to the allocations process but significantly alters traditional versions of that model. Procedural innovations are made not only in the means of establishing the amount and cost of materials relevant to an academic discipline, but also in the manner of using these figures to calculate specific allotments. More radically, need and enrollment factors, characteristically confined to usage-based formulas, are incorporated in order to modify abstract costs by probable levels of local demand.

ne of the recurring challenges of academic librarianship is the equitable division of materials budgets among rival subject areas. An active tradition of reporting practical approaches to the task, as well as a growing body of theoretical research on the topic, witnesses the seriousness with which the problem is regarded. When a Collections Advisory Committee was appointed within the Central Libraries system of the University of Cincinnati and charged with developing a formal method of distributing monograph funds, there was, consequently, a pervasive sense of entering a region well populated by competing methodologies. Amid the conflict of ideas characteristic of this territory, agreement does exist, however, on at least one point. This common theme is stated clearly in the RTSD "Guidelines for the Allocation of Library Materials Budgets": "each institution will need to develop its own method for allocation which will apply to its own circumstance." 1

Recognition of the importance of local conditions usually focuses on the more easily definable aspects of the particular institution. Less frequently acknowledged is the significance of an institution's intellectual and political climate, which always directs, and sometimes dictates, choice among different solutions to a problem. Since the Collections Advisory Committee itself was deliberately constituted to reflect the various elements of the local environment, it was sensitive to differing points of view and concerned to accommodate them. Indeed, there was a general realization that for a new allocations method to command the consensus required for smooth implementation, the technique must not only be consistent in its treatment of objective factors but also

William McPheron is subject librarian for English, American, and comparative literature, Lockwood Memorial Library, State University of New York at Buffalo. Though the paper was personally authored, the development of the formula was a joint effort. The other members of the committee whose work is presented here were: Elizabeth Douthitt Byrne, head, Design, Architecture, Art, and Planning Library; Dorice DesChene, head, ChemistryBiology Library; L. Ronald Frommeyer, head, Acquisitions Department; Sally Moffitt Neely, reference librarian and bibliographer for history; and Randall L. Roberts, reference librarian and bibliographer for the social sciences-all of the University of Cincinnati Central Libraries. 
be congruent with the subjective expectations of the librarians, faculty, and administrators involved.

From the twin necessity of methodological integrity and political suitability, there emerged a quantitative procedure for distributing monograph funds that found widespread acceptance. Its interest at large is twofold. First, it exemplifies how a medium-sized academic library, relying on a representative committee of subject specialists and the cooperation of their colleagues, successfully introduced an impersonal formula into the allocations process. Second, the procedure itself, a tripartite operation, accomplished a major revision of traditional size-of-literature approaches to the division of monograph funds. The initial stage introduces a series of refinements in determining the total cost of monographic literature relevant to academic budget lines. The second stage modifies those cost figures by incorporating need and enrollment factors characteristically excluded from the size-ofliterature model. The final stage presents a means of calculating the amount allotted to a budget line in the context of both the adjusted cost figures and prior funding, so that historical inequities relative to other lines are redressed. Tables 1 and 2 illustrate the steps of these stages for two exemplary budget lines, history and biology, and provide the structure for the account that follows.

\section{STAGE 1: ESTIMATING COST OF MONOGRAPHIC LITERATURE}

Lines $A$ through $E$ on tables 1 and 2 constitute the foundation of the allocations procedure, representing the steps by which the total cost of all relevant monographic and other nonserial materials was estimated for each budget line. This amount was figured on a five-year basis in order to compensate for any unusual, short-term fluctuations in the production and expense of library materials in a subject area.

Line A: Current Domestic Monograph Base indicates the number and retail price of monographs pertinent to a line that were published and/or commercially distributed in the United States during the years measured. This information was derived from annual subject analyses of monographs compiled by Baker \& Taylor for its domestic approval plan. Prior participation in this plan had provided both familiarity with its coverage and experience with the vendor's application of its own subject terminology. Understanding the latter was particularly important, since Baker \& Taylor's subject categories were at once different from and more numerous than local budget lines. This is a familiar dilemma with size-of-literature approaches, for the structure of external production data seldom dovetails exactly with internal accounting organization. ${ }^{2}$ To solve the problem, reliance was placed on the judgments of the subject specialists. On the basis of opinions expressed by the selectors and agreements made among them, all the vendor's categories were assigned in whole or part to one or more of the local budget lines.

Table 3 displays the products of this process for history and biology. To calculate the Current Domestic Monograph Base for either of these budget lines, the percentages shown on table 3 were applied to the costs of monographs in those subject categories as reported for that year by Baker \& Taylor. Each year's Domestic Base for every budget line was, in short, a composite figure consisting of varying portions of different subject categories. These annual amounts, entered along line $A$ on tables 1 and 2 , then functioned as the foundation on which to build an estimate of the expense of all monographic materials relevant to a budget line over the full five-year span. ${ }^{3}$ Methodologically, it should be emphasized, these figures stem from a combination of objective data and subjective judgment: verifiable information about the numbers and cost of domestic monographs was rendered locally useful by the exercise of solicited opinion. This mixture is characteristic of the allocations procedure as a whole and contributed to its positive reception.

Line B: Foreign Trade Monograph Factor estimates the cost of commercial monographs published outside the United 
TABLE 1

HISTORY

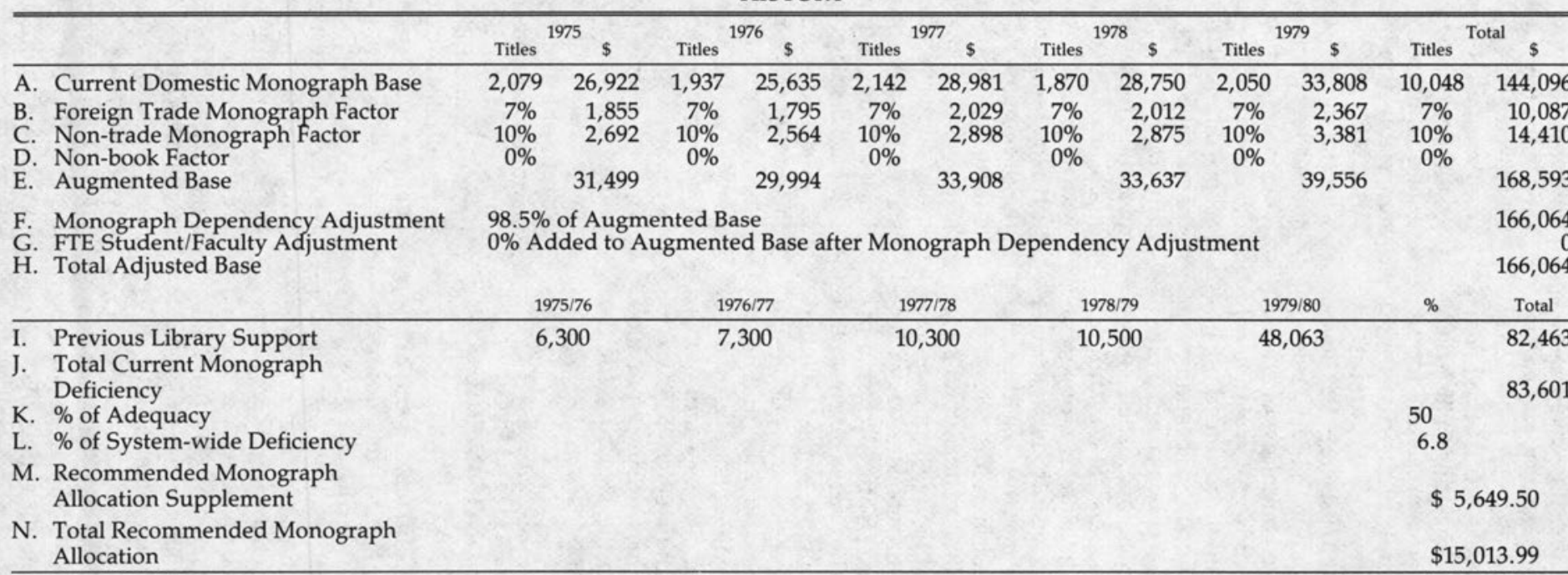


TABLE 2

BIOLOGY

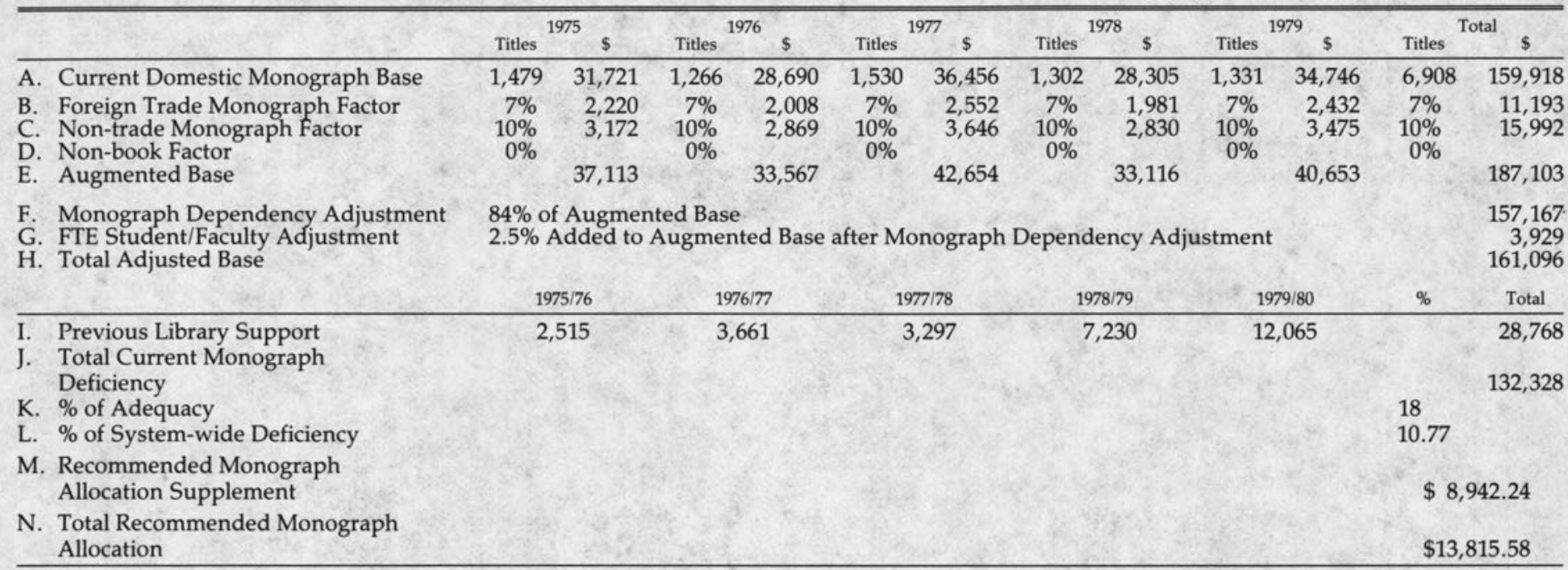


TABLE 3

DISTRIBUTION OF BAKER \& TAYLOR SUBJECT CATEGORIES

\begin{tabular}{rlrl}
\hline \multicolumn{1}{c}{ History } & & \multicolumn{1}{c}{ Biology } \\
\hline $10 \%$ & Religion & $10 \%$ & Paleontology \\
100 & History & 100 & Biology \\
95 & History of Specific Areas & 100 & Biophysics \\
100 & United States History & 75 & Biochemistry \\
50 & Auxiliary Historical Science & 100 & Microbiology \\
10 & Geography & 100 & Physiology \\
5 & Sociology & 30 & Conservation of Natural Resources \\
10 & U.S. Government & 100 & Botany \\
5 & International Relations & 100 & Zoology \\
20 & Military and Naval Sciences & 100 & Human Biology \\
10 & Labor Economics & 10 & Medicine \\
& & 5 & Internal Medicine \\
& & 5 & Pathology \\
& & 5 & Health Sciences \\
& & 5 & Neurology \\
& & 5 & Agriculture \\
& & 10 & Agronomy \\
& & 10 & Horticulture \\
& & 10 & Forestry \\
& & 10 & Animal Science \\
& & 50 & Fish Culture and Fisheries \\
& & 20 & Veterinary Medicine \\
\hline
\end{tabular}

States and not distributed domestically, by treating the expense of these foreign imprints as a percentage of a budget line's Domestic Base. Because the available subject analysis of foreign book production is not sufficiently detailed, most size-ofliterature models do not explicitly account for nondomestic monographs. Yet this neglect risks inequities, since the proportion of a literature's foreign titles varies considerably among academic disciplines. Calculating foreign costs as a percentage of domestic monograph outlays does allow direct compensation for such variations among subject areas.

The actual determination of specific percentages for particular budget lines was based on a series of considerations. The relative importance of foreign monographs in a discipline's literature was established on the basis of subject specialists' judgments. These were elicited through a questionnaire which asked selectors to rate the centrality of the major classes of monographic materials to their fields. Analysis of answers regarding foreign imprints resulted in the placement of every budget line in one of four general levels of dependency. Then, to ascertain the percentages allotted to each of these general levels, the proportions of local monograph budgets actually expended in recent years on foreign monographs were examined to identify ranges corresponding to the levels, and the median of each of these ranges was then taken as a representative figure. Finally, a number of discipline-oriented citation studies, which included data on the frequency of reference to foreign language monographs, were consulted to countercheck the median figures derived from internal acquisitions statistics. ${ }^{5}$ Emerging from this process were 0 percent, 2 percent, 7 percent, and 22 percent as the portions of the Domestic Base that would be added as the Foreign Trade Monograph Factor at each year along line $B$ for budget lines at the four different levels of dependency.

These specific figures possess, of course, no external applicability, since they are products of the subjective judgments of particular selectors and local acquisitions data. But the method itself does have independent value as a consistent means of registering variations in the importance of foreign monographs. It also has the advantage of respecting the major differences in the cost of monographs among the disciplines, as tables 1 and 2 il- 
lustrate. ${ }^{6}$ Both history and biology qualified for a 7 percent foreign imprint supplement; but while the number of domestic titles in biology over the five-year period is only 70 percent that of history, because of the greater expense of biological monographs, the total amount of its Foreign Trade Monograph Factor was actually 10 percent higher than the one for history.

Line C: Non-Trade Monograph Factor estimates the cost of those monographic imprints, both domestic and foreign, that are unavailable through normal commercial channels, by again calculating their expense as a percentage of the Domestic Base. This category includes not only monographs published by learned societies and professional associations but also nonserial, nondepository titles produced by governmental and other official agencies which must be purchased from monograph funds. Such materials are typically acquired directly from the issuing body and consequently are not usually included in approval plan statistics, such as those from Baker \& Taylor. Nor are there other sources of production data that would allow direct measurement of the cost of this type of monograph in specific subject areas. Yet this kind of publication does play a major role in some disciplines, and failure to account for it in a size-of-literature model threatens to penalize those budget lines. By computing the expense of noncommercial monographs as a variable percentage of the Domestic Base, different degrees of need for such publications are explicitly recognized.

Arriving at the actual percentages repeated the technique used for the Foreign Trade Factor and again involved the evaluation of subject specialists' ratings, internal acquisitions records, and external citation studies. From analysis of this information came 20 percent and 10 percent as the portions of the Domestic Base added along line $C$ for budget lines with respectively heavy and moderate reliance on nontrade publications. The applied sciences and business were the recipients of the higher supplement, with many of the pure sciences and the social sciences qualifying for lower compensation. Disciplines in the humanities reported virtually no re- liance on this material.

Line D: Non-Book Factor is designed to register the cost of another type of library resource that is often purchased for subject areas from their allocated monograph funds but is seldom explicitly acknowledged in size-of-literature approaches. This nonbook category encompasses the complete gamut of print and nonprint formats, but specifically excludes microform reproductions of previously published texts, since these are properly an aspect of retrospective collection development and hence not germane to measuring the total expense of current nonserial production. In the absence of statistics sufficiently detailed to allow direct calculation of nonbook costs to individual disciplines, a percentage of a budget line's Domestic Base was again employed to ascertain the amount of the supplementary Non-Book Factor. The assignment of exact rates of compensation was, in practice, simplified by the fact that subject specialists reported only one area with a significant need for nonbook materials. The actual percentage of monograph funds expended on such resources by that budget line in the preceding year was consequently adopted and used to calculate its Non-Book Factor from each year's Domestic Base along line $D$. No attempt was made to construct representative figures, as had been done for the Foreign Trade Monograph Factor and the Non-Trade Monograph Factor. Local circumstances in which more emphasis is placed on nonbook materials would, of course, require greater methodological rigor in determining each line's Non-Book Factor.

One general aspect of this methodology as it applies not only to the Non-Book Factor but also to the Non-Trade Monograph Factor is problematic and deserves a cautionary notice. By calculating these supplements as percentages of the Domestic Base, the cost differentials of domestic trade monographs among the various subject areas are perpetuated. This is an advantage in computing the Foreign Trade Monograph Factor, since the prices of domestic and foreign imprints parallel each other; but there is no confirmation of this pattern for nonbook and nontrade items. In the absence of evidence that, for example, a 
technical report or videocassette in biology mirrors domestic book prices and so costs approximately 60 percent more than comparable materials in history, the danger exists of overestimating the expense of nonbook and nontrade resources for some budget lines and underestimating it for others.

Line E: Augmented Base is the sum of the Domestic Base and its foreign, nontrade, and nonbook supplements. This is entered on tables 1 and 2 for each year monitored as well as for the entire period. The total amount represents an exhaustive budget, theoretically sufficient to acquire all monographic and nonserial resources published during that span of time. This comprehensive figure is the critical one for subsequent stages of the allocations procedure.

\section{STAGE 2: ACCOUNTING FOR LOCAL CIRCUMSTANCES}

Lines $F$ through $H$ adjust the exhaustive Augmented Base in light of curricular and enrollment factors specific to the individual institution. Size-of-literature approaches do not typically assess the instructional and research orientations of local curricula in order to estimate what portion of the total body of relevant materials is actually needed for their support. And only occasionally is this allocations model made responsive to differences in the size of student and faculty populations among subject areas. Yet the particular nature of a discipline's local organization as well as the number of people active in its program can significantly affect its practical requirements for monographic resources. The importance of such pragmatic demand in making allocations decisions is, of course, the central premise of a variety of recently developed usage-based models for distributing materials budgets. ${ }^{7}$ Their emphasis on usage as the primary criterion for allotting funds enters this allocations procedure in stage two as a secondary element. Here, measurements reflective of probable demand act to modify each budget line's Augmented Base.

Line F: Monograph Dependency Adjustment estimates the percentage of the Augmented Base that is required to support a discipline as it is locally organized. This figure establishes the extent of a program's need for its full range of monographic resources and thus reflects both the nature of the local curriculum and the orientation of the field at large. Resistance among selectors and their faculties to such a quantified judgment is understandable, since it entails acknowledgment of limits and a retreat from an ideal standard of support. But in the absence of abundant funding, it is important to recognize that degrees of dependence on monographic literature do vary among subject areas.

The Monograph Dependency Adjustment serves to account for these differences by establishing the minimum percentage of the Augmented Base necessary for adequate support. To determine the size of this figure for a budget line, responses to the collections questionnaire were again used. Subject specialists' ratings of the dependence of their discipline on current monographic resources were tallied for every line. These totals clustered into five groups, and the median number of points scored within each group was treated as representative. This median number was then translated into a percentage and became a group's Monograph Dependency Adjustment. For example, history fell into the group that registered 82.7 of the maximum 84 points possible on the questionnaire, which yields the 98.5 percent of its Augmented Base brought forward on line F; while biology, a member of the group with a median of 70.6 points, was allowed only 84 percent of its ideal monographic budget.

These figures are indicative of local circumstances only, but what can claim general applicability is the concept of introducing projected levels of monographic need into a size-of-literature model by employing these estimates to variably reduce the total costs of disciplines' literature bases. Also of general use is the technique of relying on selectors' professional perception of the orientation of local programs to establish such projections. Though operating without objective data, this technique does inject the specialists' personal knowledge into the allocations procedure at a particularly critical point 
and consequently provides a basis on which anxiety about quantifying the process can be shared and resistance to it allayed.

Line G: FTE Student/Faculty Adjustment responds to that type of demand for monographs which is generated by large numbers of people in a program. This kind of usage is presumed to be intense but narrow, justifying the duplication of titles central to a discipline but not warranting the broadening of its literature base as delimited by the Monograph Dependency Adjustment. Since its intent is to provide supplementary support for core works, the Student/Faculty Adjustment comes after the reduction of the Augmented Base on line $F$ and is calculated as a percentage of that line.

To identify those disciplines receiving compensation at this point, the number of full-time equivalent (FTE) students and faculty during the preceding five years was compiled for each program. Analysis of this data disclosed four groups of heavy concentration, into one of which about a half of the budget lines fell, the others not showing sufficient density to warrant this kind of support. On the basis of local estimates of reasonable rates of duplication, supplementary amounts of 10 percent, 7.5 percent, 5 percent, and 2.5 percent of their reduced Augmented Bases were granted budget lines in the respective groups. Tables 1 and 2 illustrate this operation, with biology receiving the minimum compensation for high enrollment and history not qualifying for any supplement.

Restricting use of enrollment information to predicting demand for duplication is a characteristic principle of size-ofliterature models. Less typical is the integration of such statistics directly into the formula in order to adjust the cost of a discipline's base. ${ }^{8}$ Yet in some programs the need for multiple copies is clearly greater than in others, and this higher demand does increase, in effect, the overall expense of their monographic requirements. It is this fact that justifies the Student/Faculty Adjustment.

Line H: Total Adjusted Base is the sum of a budget line's enrollment supplement and its Augmented Base after reduction by the
Monograph Dependency Factor. This amount represents the minimally adequate monographic budget over the fiveyear period for a discipline as it is locally practiced. It is this figure that is used in the final stage of the allocations procedure.

\section{STAGE 3: COMPUTING THE ALLOCATIONS}

Lines I through $N$ are the steps by which the Total Adjusted Base is translated into specific allocation recommendations. The salient feature of this stage is the use of prior levels of monographic funding as the context for computing each budget line's allotment. Advocates of formulas, whether based on usage or literature size, consistently warn against incorporating such historical precedence, since these earlier budgetary decisions are seldom the products of a rationalized process. While this position commands assent in the abstract, it neglects the practical and political problems posed by concern about funding inequities in the recent past. Where significant imbalances are perceived to have developed in the immediately preceding years, a formula cannot start afresh but must take account of prior funding if it is to correct these inherited discrepancies. The perception of inequities is not uncommon and the need to deal effectively with such a situation prompted the technique of stage three, which allows the calculation of present allocations to account for past practices and compensate systematically for them.

Line I: Previous Library Support records the amounts available to a budget line for the acquisition of current monographic resources during each of the years monitored, as well as for the period as a whole. Monies budgeted for retrospective materials are excluded here, since the purpose is to gauge a line's capacity to purchase the body of literature represented by its Total Adjusted Base.

Line J: Total Current Monograph Deficiency is the difference between line $I$, a discipline's actual funding over the period, and line $H$, the estimate of its minimally adequate monograph budget for those years. This deficit measures the distance between the needs of the local program 
and the support provided.

Line K: \% of Adequacy parallels line J; it also compares actual funding with needed funding but presents this relationship proportionally, as the percentage of the locally appropriate budget that was, in fact, furnished. It is at this point that any inequities in earlier allocation patterns will emerge, since major differences in percentages here indicate that the requirements of some disciplines were being met more fully than others. Such an imbalance is evident in tables 1 and 2, with the Adjusted Bases of history and biology being approximately equal, but their levels of recent support varying dramatically. The result on line $K$ is a 50 percent adequacy rate for history and only 18 percent for biology.

Line L: \% of System-Wide Deficiency represents a single budget line's share of the cost of all locally needed but unacquired monographic resources over the period measured. This percentage is a special version of the figure conventionally employed by size-of-literature models to distribute funds. In that approach, a subject area's allotment typically depends on the cost of its literature relative to the expense of the cumulated bases of every field. In this procedure, a discipline's previous support is first deducted from its Adjusted Base and entered at line J as its Current Deficiency. It is, then, the cost of this remaining, unacquired segment relative to the cost of the unacquired segments for all disciplines that determines the division of funds. The greater the discrepancy between a budget line's needed and its actual funding during the previous years, the larger its share of the body of materials still required by the libraries, and hence the higher its portion of present funds. Thus, by making a line's allocation reflect both the cost of its necessary materials and the level of its past support, inequities that occurred in the immediately preceding period are redressed.

This compensatory process emerges clearly on the accompanying tables. The libraries' total of unacquired monographic resources, the sum of every discipline's line $J$, amounted to $\$ 1,228,713$. History was unable to purchase $\$ 83,601$ of its ma- terials, which constituted 6.8 percent of the libraries' unfilled needs, while biology's shortfall of $\$ 132,328$ was 10.77 percent of the system's overall requirements. Although their Adjusted Bases are almost identical, because of its relatively lower funding in prior years, biology thus received a 3.97 percent higher allocation than history.

Line M: Recommended Monograph Allocation Supplement reflects a further incorporation of historical precedent. Instead of distributing the libraries' full monograph budget according to the percentages of need entered on line $L$, these figures were applied only to new monies. The resulting amounts were then used to supplement each discipline's allotment from the preceding year, historical funding decisions thus being substantially maintained.

This limited use of the percentages produced by the allocations procedure is not inherent in the procedure itself but was recommended by local considerations. Its effect was to avoid the precipitous reallocation of funds which would have occurred had the formula's figures been allowed to operate on the whole monograph budget. For example, biology's 10.77 percent share would have yielded a steep rise to $\$ 22,706$, an upward jump of 88 percent over its prior year's allotment of $\$ 12,065$. Such sudden increases inevitably entail parallel reductions in other budget lines. Even in conditions of abundant funding, this is a difficult move; when there are surpluses nowhere, it can jeopardize acceptance of a quantified approach to the materials budget. In local circumstances where this concern is less prominent, it might be unnecessary to slow the pace at which reassignment of support among subject areas is effected.

Line N: Total Recommended Monograph Allocation is the sum of a discipline's share of new monies, as calculated on line $M$, and the amount of its allocation from the preceding year, as established by prior practice. This final figure is a compromise, superimposing the results of present quantification on past informality. This does, however, provide continuity and consequently assures a smoother transition to the new allocations method. 


\section{LIMITATIONS OF THE PROCEDURE}

This allocations procedure suffers from several methodological shortcomings that ought to be noted.

1 . No provision is made for the preferential consideration of programs accorded high priority. Because disciplines are treated equally within the framework of the formula, a portion of the monograph budget must be reserved for administrative assignment to areas requiring special support.

2. The mixture of objective data and subjective judgment employed in the procedure renders the accuracy of its results questionable. The inevitable imprecision caused by reliance on selectors' opinions is tolerable initially, since their participation in developing the formula encouraged its positive reception. After this introductory phase, more rigorous data is preferable. This does not, however, mean that staff involvement ceases. Indeed, local use and citation studies may provide the best objective information about a discipline's actual dependency on different types of monographic resources, and responsibility for designing and executing these studies resides naturally with the subject specialists.

3 . Focus on current monographic resources ignores the need for out-of-print and antiquarian titles. Though determining "retrospective acquisitions rates" is noted in the RTSD "Guidelines" as a problem "not yet handled by any library budget formula, " it is susceptible to solution within this procedure. For retrospective funding could be handled as a supplement to a budget line's Domestic Base and, like the other factors in stage one, calculated as a percentage of that base. Computing a line's percentage would require both a numerical measure of a discipline's reliance on retrospective monographs and a statistical account of the adequacy of existing retrospective holdings. The latter figure would serve to define that portion of the universe of retrospective titles presumed to be absent from the collection, while the degree of dependency would establish the amount of those lacking resources which ought still to be acquired. Multiplying the rate of reliance against the proportion of materials required would then yield the relative percentage of a discipline's need for retrospective support. For example, if 80 percent of history's monograph dependency is retrospective-a possibility suggested by a recent study $^{10}$-and evaluation of local resources shows 40 percent of retrospective titles absent, then the product of these figures, or 32 percent, becomes the portion of history's Domestic Base that represents its need for out-of-print funding. Its Augmented Base would be increased by this amount before the adjustments of stage two are made.

\section{CONCLUSION}

At the end of his survey of techniques for distributing academic book budgets, Jasper G. Schad recommends assigning the staff responsible for collection development "the task of preparing an allocation for review by the library administration." issued from such an assignment and is informed by the attitudes of librarians who daily encounter collection problems familiar to most medium-sized university libraries. The manner of the procedure is eclectic, reflecting the concerns of different disciplines as well as drawing concepts from competing allocation models. Its method is pragmatic, relating general principles of allocation theory to particular circumstances. Though lacking the formal elegance of a mathematical formula, the procedure does possess an unusual inclusiveness of considerations relevant to the allotment of monograph funds. This is a sign of its roots in actual practice and may be the source of its greatest interest.

\section{REFERENCES AND NOTES}

1. "Guidelines for the Allocation of Library Materials Budgets," in David L. Perkins, ed., Guidelines for Collection Development (Chicago: American Library Assn., 1979), p.33. Jasper G. Schad provides 
a convenient survey and critique of published allocations formulas in "Allocating Materials Budgets in Institutions of Higher Learning," The Journal of Academic Librarianship 3:328-32 (Jan. 1978).

2. The sources of publishing data employed by different practitioners of the size-of-literature approach have varied widely. William M. Randall, "The College-Library Book Budget," Library Quarterly 1:421-35 (Oct. 1931) relied on a printed bibliography, Charles B. Shaw's List of Books for College Libraries (1st ed., 1930). William E. McGrath, "Determining and Allocating Book Funds for Current Domestic Buying," College \& Research Libraries 28:269-72 (July 1967) analyzed an annual cumulated volume of the American Book Publishing Record, BPR. Bette Dillehay, "Book Budget Allocation: Subjective or Objective Approach," Special Libraries 62:509-14 (Dec. 1971), examined book reviews appearing in major discipline journals, while more recently Richard Hume Werking and Charles M. Getchell, Jr., "Using Choice as a Mechanism for Allocating Book Funds in an Academic Library," College \& Research Libraries 42:134-38 (March 1981), have demonstrated the utility of a single, general reviewing organ. All these involved the problem of relating external information to internal budget-units, and in each case it was resolved by the exercise of informed judgment.

3. Budget lines not identified with academic departments, such as reference and documents, were handled outside the allocations procedure because cost data was not available. For lines with a primary dependence on foreign monographs, such as German and Romance languages, the Domestic Base was constructed from expenditure figures provided by university libraries collecting comprehensively in the fields.

4. Randall, "College-Library Book Budget," Dillehay, "Book Budget Allocation," and Werking and Getchell, "Using Choice," all appear implicitly to assume that foreign titles are represented in appropriate proportions in the bases they variously employ. McGrath, "Determining and Allocating Book Funds," p.271, does observe that foreign imprints present a special problem and suggests that they "be handled on an ad hoc basis."

5. Representative citation rates of foreign language imprints are the following: Herman $\mathrm{H}$. Fussler, "Characteristics of the Research Literature Used by Chemists and Physicists in the United States," Library Quarterly 19:128-29 (April 1949) reported 11.8 percent for chemistry and 37.1 percent for physics; Shirley A. Fitzgibbon's convenient survey article, "Citation Analysis in the Social Sciences," in Robert D. Stueart and George B. Miller, Jr., eds., Collection Development in Libraries: A Treatise, Part B (Greenwich, Conn.: JAI Press, 1980), p. 291-344, records a range from a high 15 percent (political science) to no use (business administration and education); Richard Heinzkill, "Characteristics of References in Selected Scholarly English Journals," Library Quarterly 50:361-63 (July 1980) showed 9 percent.

6. Actually, if the figures for the United States, the United Kingdom, and the Federal Republic of Germany provided by The Bowker Annual of Library and Book Trade Information (26th ed.; New York: Bowker, 1981), p.344-45, 351, 352, are analyzed, American hardcover books in the sciences cost approximately 70 percent more than ones in history, biography, and travel. German scientific texts run, however, about 170 percent more than their historical and geographical counterparts, while the analogous rate for British books is an even higher 180 percent. If the bases from which these figures derive are truly comparable, it may be that an additional adjustment within the Foreign Trade Monograph Factor is necessary to compensate for steeply higher ratios of differences between foreign book costs by discipline.

7. Most recent work on allocations models has emphasized usage, measured and weighted in various ways. S. K. Goyal, "Allocation of Library Funds to Different Departments of a University-An Operational Research Approach," College \& Research Libraries 34:219-22 (May 1973), stresses numbers of faculty and students; William E. McGrath, "A Pragmatic Allocation Formula for Academic and Public Libraries with a Test for Its Effectiveness," Library Resources \& Technical Services 19:356-68 (Fall 1975), relies on circulation figures by subject and average cost of books by discipline; Steven D. Gold, "Allocating the Book Budget: An Economic Model," College \& Research Libraries 36:397-402 (Sept. 1975), employs the number of student credit hours in an academic department, weighted both by extent of library work required and putative value of the program to the university; R. J. Welwood, "Book Budget Allocation: An Objective Formula for the Small Academic Library," Canadian Library Journal 34:213-19 (June 1977), uses three variables-enrollment, circulation, and number of courses-weighted by cost differences among disciplines; Thomas J. Pierce, "An Empirical Approach to the Allocation of the University Book Budget," Collection Management 2:39-58 (Spring 1978), focuses on size of departments, local availability of resources, subject characteristics or library orientation of disciplines, and relative price of materials. Schad, "Allocating Materials Budgets," p.329-330, criticizes the basic premise of these models that use in an academic library is predictive of need.

8. Randall, "College-Library Book Budget," p.421, states the standard position that "the number of 
students enrolled" is important "chiefly with respect to duplicates required for collateral reading." Where size-of-literature models do take enrollment into account, for example, Eleanor W. Falley, "An Impersonal Division of the College Book Fund," Library Journal 64:933-35 (Dec. 1, 1939) and Don Revill, "A Book Fund Allocation Formula," New Library World 75:162-63 (Aug. 1974), the factor is employed to predict overall demand, not duplication needs.

9. Guidelines for Collection Development, p.41.

10. Clyve Jones, Michael Chapman, and Pamela Carr Woods, "The Characteristics of Literature Used by Historians," Journal of Librarianship 4:147-49 (July 1972).

11. Schad, "Allocating Materials Budgets," p.331.

\section{APPLICATIONS INVITED FOR EDITOR OF COLLEGE \& RESEARCH LIBRARIES}

Nominations and applications are invited for the postion of editor of College $\mathcal{E}$ Research Libraries. The editor is appointed for a three-year term which may be renewed for an additional three years. Applicants must be members of ALA and ACRL. Qualifications include experience in academic libraries, evidence of research and editing activity, and a broad knowledge of the issues confronting academic libraries.

A small honorarium for the editor and funding arrangements for editorial assistance are available.

Nominations or résumés with the names of three references may be sent to Larry Wilt, Chair, College \& Research Libraries Search Committee, Albin O. Kuhn Library and Gallery, University of Maryland Baltimore County, Catonsville, MD 21228. The deadline for applications is May 20, 1983. 